\title{
Разработка методов численного анализа динамики функциональной активности спинного мозга у пациентов, перенесших осложненную спинальную травму
}

\author{
Ю. Е. Педаченко, А. С. Нехлопочин \\ Институт нейрохирургии имени академика А. П. Ромоданова НАМН Украины, г. Киев

\section{Elaboration of methods of numerical analysis of dynamics of the spinal cord functional activity in patients, suffering complicated spinal trauma}

\author{
Yu. E. Pedachenko, A. S. Nekhlopochin
}

Romodanov Institute of Neurosurgery, Kyiv

\section{Реферат}

Цель. Разработка методов численного анализа оценки неврологического статуса пациентов, перенесших позвоночно-спинномозговую травму (ПСМП).

Материалы и методы. На основании клинических наблюдений рассмотрены возможные варианты математической интерпретации динамики восстановления неврологического статуса и регресса неврологических расстройств.

Результаты. Разработаны методы расчета параметров «Динамика восстановления неврологического статуса» и «Динамика восстановления неврологических расстройств», характеризующих различные сферы регресса функционального дефицита у пациентов с травматическим поражением спинного мозга (СМ).

Выводы. Предложенные методы численного анализа могут использоваться для определения целесообразности и эффективности различных методов хирургической и медикаментозной коррекции у пациентов с ПСМП.

Ключевые слова: неврологический статус; травма спинного мозга; численный анализ; динамика восстановления.

Abstract

Objective. Elaboration of methods of numerical analysis, concerning estimation of neurological status in patients, suffering consequences of vertebra-spinal trauma (ПСМП).

Materials and methods. Basing on clinical observations, possible variants of mathematical interpretation of the restoration dynamics of neurological status and the neurological disorders regress were considered.

Results. The methods of the parameters calculation «Dynamics of the neural status restoration» and «Dynamics restoration in neurological disorders», which characterizes various spheres of the functional deficiency regress in patients, suffering traumatic affection of spinal cord, were elaborated.

Conclusion. The proposed methods of numerical analysis may be used for determination of expediency and efficacy of various methods of surgical and medicinal correction in patients with traumatic vertebro-spinal affection.

Keywords: neurological status; spinal trauma; numerical analysis; dynamics of restoration.

Травматическое повреждение СМ является одним из наиболее тяжелых последствий механического воздействия на организм человека и характеризуется стабильно высокой летальностью в острый период травмы, стойкими ограничениями жизнедеятельности и утратой трудоспособности в отдаленном периоде. По данным ВО3, в мире около 2,5 млн. человек, средний возраст которых составляет 33,4 года, страдают от последствий перенесенной травмы СМ.

Согласно данным International Campaign for Cures of Spinal Cord Injury Paralysis только в четырех странах США, Канаде, Австралии и Англии - ежегодные расходы на медицинское обеспечение пациентов с последствиями ПСМТ превышают 10 млн. долларов. Последнее десятилетие характеризуется постоянным увеличением частоты ПСМТ, которое является наибольшим в странах с низкими доходами населения. Внедрение в практическое здравоохранение унифицированных протоколов оказания экстренной помощи, современных методов нейровизуализации, оптимизированных методов анестезиологического пособия и хирургического лечения позволили в значительной степени снизить летальность у данной категории пострадавших, что закономерно привело к увеличению количества пациентов с травматической болезнью СМ, нуждающихся в усовершенствованных методах лечения на всех этапах ее развития.

Опубликованы результаты значительного количества исследований, направленных на разработку методов минимизации последствий ПСМТ, включающих хирургическое, фармакологическое и физическое воздействие.

Закономерно предложено множество систем оценки эффективности проводимой терапии.

На основании анализа данных литературы нами выделены следующие методы оценки регресса последствий ПСМТ: 
клинико-неврологические - международные стандарты неврологической классификации травмы CM (International Standards for Neurological Classification of Spinal Cord Injury - ISNCSCI), стандарты оценки Американской ассоциации проблем повреждения позвоночника (American Spinal Injury Association - ASIA) и др. [1 - 3];

электронейрофизиологические - двигательные и чувствительные вызванные потенциалы, функциональная нейрофизиологическая оценка и др. [4 - 6];

основанные на нейровизуализации - магнитно-резонансная томография, функциональная магнитно-резонансная томография и др. [7 - 9];

функциональной самооценки: тест 6- и 10-минутной ходьбы, шкала баланса Берга, шкала ограничения качества жизни и др. [10 - 12].

Наиболее широко используются, особенно в остром периоде ПСМТ, клинико-неврологические шкалы. «Золотым стандартом» признана шкала ISNCSCI, преимущества которой неоспоримы: простота оценки, основанная на классических принципах описания неврологического статуса, высокая детализация, сопоставимость результатов в динамике.

Однако эта шкала имеет ряд существенных недостатков, один из которых - отсутствие математического алгоритма оценки эффективности применяемых методов лечения у пациентов с различным уровнем поражения CM, из-за чего ее применение в значительной мере лимитируется и фактически ограничивается 5-буквенной шкалой ASIA.

Цель исследования: разработка метода численного анализа результатов оценки неврологического статуса по шкале ISNCSCI для сравнения эффективности терапии у пациентов с различным уровнем поражения CM.

\section{Материалы и методы исследования}

Проанализированы данные литературы для определения существующих методов численной интерпретации результатов тестирования по шкале ISNCSCI. Ha ocновании изучения клинической картины у пациентов с травмой позвоночника, сочетающейся с поражением CM, рассмотрены варианты математической интерпретации динамики восстановления неврологического статуса и регресса неврологических расстройств.

Система оценки неврологического статуса ISNCSCI, утвержденная в 1992 г. Американской ассоциацией спинальной травмы, это четко стандартизированный алгоритм действий, направленный на определение уровня и степени повреждения СМ. Обследование пациента проводится в положении лежа. Последовательно сверху вниз оценивается тактильная и болевая чувствительность. Предусмотрен анализ 28 дерматомов по схеме, изображенной на стандартном бланке ISNCSCI, с цифровым отображением результата тестирования на каждом уровне. Возможны три варианта оценки сенсорной функции: 0 - отсутствие чувствительности, 1 - измененная (пониженная либо повышенная) чувствительность, 2 - нормальная чувствительность. Когда пациент не может дифференцировать касание и укол, подразумевается отсутствие болевой чувствительности. Когда проверка чувствительности в определенной зоне не представляется возможной (выраженный болевой синдром, иммобилизация, ампутация либо контрактура, нарушающая более 50\% объема движений), используется аббревиатура NT. Двигательная функция оценивается с двух сторон по 10 ключевым группам мышц с определением одного из 6 вариантов согласно шкале Медицинского исследовательского совета (шкала MRC) [13]: 0 баллов
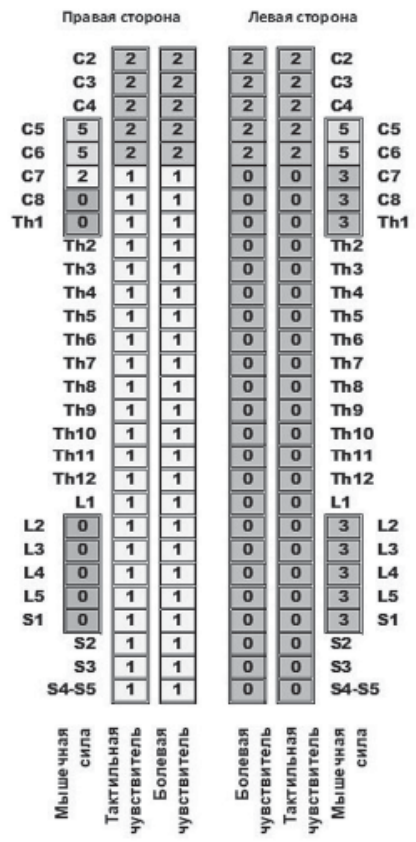

Рис. 1. Оценка неврологического статуса пачиента с травиой шейного отдела позвоночника, осложненной повреждением СМ.

A - при госпитализащии, Б - через б мес после оперативного вмешательства.ASIAD.
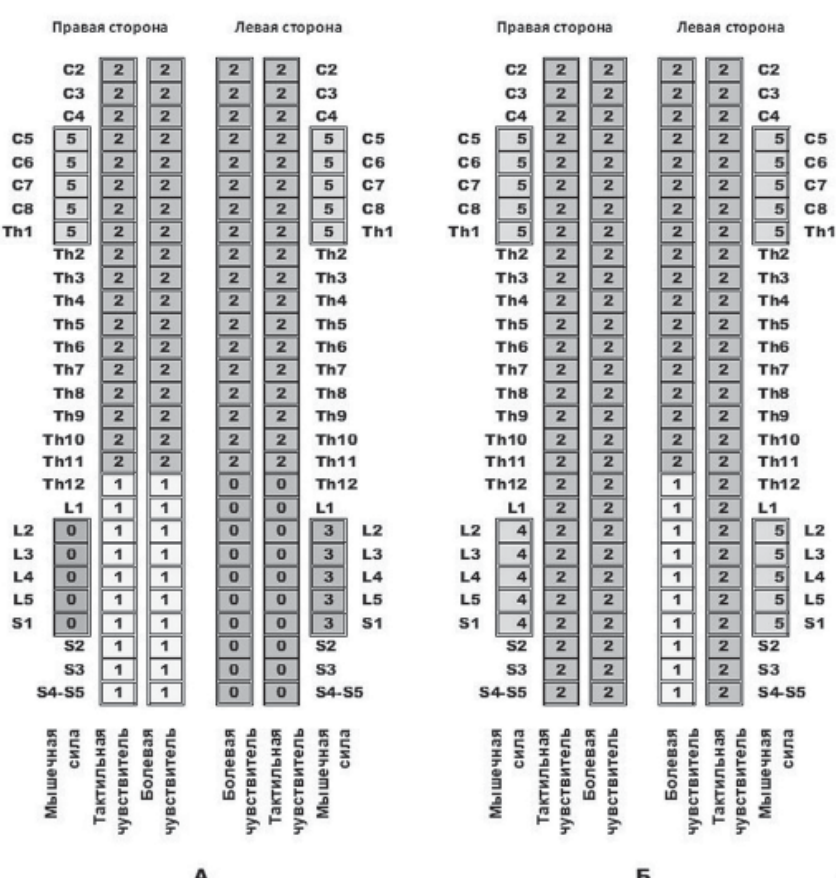

Рис. 2. Оценка неврологического статуса пащиента с травмой нижнегрудного отдела позвоночника, осложненной повреждением СМ. А- при госпитализащии,

Б - через б мес после оперативного вмешательства. ASLA D. 
- полный паралич; 1 балл - пальпируемые или видимые сокращения мышц; 2 балла - активные движения в полном объеме без преодоления силы тяжести; 3 балла - активные движения в полном объеме с преодолением силы тяжести; 4 балла - активные движения в полном объеме с преодолением силы тяжести и небольшого внешнего противодействия; 5 баллов - активные движения в полном объеме с преодолением силы тяжести и максимального внешнего противодействия (нормальные).

Максимальная сумма баллов при условии оценки всех предусмотренных методикой дерматомов для чувствительной сферы (ЧС) - 224, для двигательной сферы (ДС) $-100$.

\section{Результаты}

Анализ алгоритмов цифровой обработки существующих систем оценки неврологического статуса не выявил схем, позволяющих сравнить динамику регресса расстройств неврологических функций у пациентов с различным уровнем поражения CM. Шкала ASIA - единственная широко распространенная система оценки, которая используется в большинстве современных исследований [14]. Следует отметить, что по шкале ASIA переход из уровня А в другие подгруппы либо переход в уровень Е из других подгрупп не имеет сложностей в оценке изменения неврологического статуса пострадавших и вариантов трактовки, но для всех других разновидностей динамики, например для перехода из ASIA B в ASIA C, возможно значительное количество клинико-неврологических вариаций. Соответственно минимальные изменения, имеющие, однако, существенное значение как для функциональной активности пациента, так и для оценки эффективности терапии, в процессе статистической обработки в связи с недостаточными разрешающими возможностями методик оценки упускаются.
Нами проведена оценка неврологического статуса пациента с травмой шейного отдела позвоночника, осложненной повреждением СМ, при госпитализации (рис. 1A). Неврологическая картина соответствовала «неполному синдрому Броун-Секара». Через 6 мес после проведенного хирургического лечения наблюдался значительный регресс как двигательных, так и чувствительных расстройств (рис. 1Б), однако при оценке статуса по шкале ASIA динамика отсутствовала.

Цифровая интерпретация оценки неврологического статуса по шкале ISNCSCI у пациента с травмой шейного отдела позвоночника, осложненной повреждением СМ, приведена в табл. 1.

Разница сумм баллов, приведенная в табл. 1, является разницей между конечным и начальным значениями анализируемого показателя и фактически отражает динамику восстановления неврологического статуса (ДС либо ЧС). В относительных величинах динамика восстановления неврологического статуса составила 48\% - для ДС и 51,3\% - для ЧС.

Рассчитывали относительные величины по формуле:

Восстановление ДС = сумма баллов ДС после операции сумма баллов ДС до операции/максимальная сумма баллов $\times 100 \%$.

Аналогичным образом определяли динамику восстановления ЧС.

Проведен также расчет оценки неврологического статуса у пациента с травмой нижнегрудного отдела позвоночника, осложненной повреждением СМ (табл. 2).

Данные в табл. 2 соответствуют схеме распределения неврологических нарушений по шкале ISNCSCI, приведенной на рис. 2.

Несмотря на значимую положительную динамику, неврологический статус пациента и непосредственно после травмы (рис. $2 A$ ) и через 6 мес после оперативного вмешательства (puc. 2Б) соответствовал ASIA D.

\begin{tabular}{|c|c|c|c|c|c|c|c|c|}
\hline \multirow{2}{*}{ Тип } & \multirow{2}{*}{ Показатель } & \multicolumn{3}{|c|}{ До операции, баллы } & \multicolumn{3}{|c|}{ Через 6 мес после операции, баллы } & \multirow{2}{*}{$\begin{array}{l}\text { Разница сумм } \\
\text { баллов }\end{array}$} \\
\hline & & справа & слева & сумма & справа & слева & сумма & \\
\hline \multirow{2}{*}{ ДС } & UEMS & 12 & 19 & \multirow{2}{*}{46} & 24 & 25 & \multirow{2}{*}{94} & \multirow{2}{*}{48} \\
\hline & LEMS & 0 & 15 & & 20 & 25 & & \\
\hline \multirow{2}{*}{ ЧС } & $\begin{array}{c}\text { Тактильная } \\
\text { чувствительность }\end{array}$ & 33 & 10 & \multirow{2}{*}{86} & 56 & 33 & \multirow{2}{*}{201} & \multirow{2}{*}{115} \\
\hline & $\begin{array}{c}\text { Болевая } \\
\text { чувствительность }\end{array}$ & 33 & 10 & & 56 & 56 & & \\
\hline
\end{tabular}

\begin{tabular}{|c|c|c|c|c|c|c|c|c|}
\hline Таблиь & 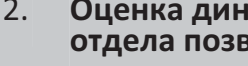 & $\begin{array}{l}\text { ики из } \\
\text { очника }\end{array}$ & $\begin{array}{l}\text { ия не } \\
\text { жнен }\end{array}$ & $\begin{array}{l}\text { ическс } \\
\text { врежд }\end{array}$ & $\begin{array}{l}\text { ryca y } \\
\text { CM }\end{array}$ & 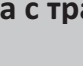 & imi & 10ro \\
\hline \multirow{2}{*}{ Тип } & \multirow{2}{*}{ Показатель } & \multicolumn{3}{|c|}{ До операции, баллы } & \multicolumn{3}{|c|}{ Через 6 мес после операции, баллы } & \multirow{2}{*}{$\begin{array}{c}\text { Разница сумм } \\
\text { баллов }\end{array}$} \\
\hline & & справа & слева & сумма & справа & слева & сумма & \\
\hline \multirow{2}{*}{ ДС } & UEMS & 25 & 25 & \multirow{2}{*}{65} & 25 & 25 & \multirow{2}{*}{95} & \multirow{2}{*}{30} \\
\hline & LEMS & 0 & 15 & & 20 & 25 & & \\
\hline \multirow{2}{*}{ पC } & $\begin{array}{c}\text { Тактильная } \\
\text { чувствительность }\end{array}$ & 46 & 36 & \multirow{2}{*}{164} & 56 & 46 & \multirow{2}{*}{214} & \multirow{2}{*}{50} \\
\hline & $\begin{array}{c}\text { Болевая } \\
\text { чувствительность }\end{array}$ & 46 & 36 & & 56 & 56 & & \\
\hline
\end{tabular}




\begin{tabular}{|c|c|c|c|c|c|c|}
\hline Таблица 3. & $\begin{array}{l}\text { Значения } \\
\text { пациенто }\end{array}$ & для сегментов & полож & а уровне и & поражения, у & \\
\hline \multirow{3}{*}{ Тип } & \multicolumn{3}{|c|}{ Пациент с травмой шейного отдела позвоночника } & \multicolumn{3}{|c|}{ Пациент с травмой нижнегрудного отдела позвоночника } \\
\hline & \multicolumn{2}{|c|}{ СФА, баллы } & \multirow[b]{2}{*}{$\triangle C \Phi A$} & \multicolumn{2}{|c|}{ СФА, баллы } & \multirow[b]{2}{*}{$\triangle \mathrm{CФA}$} \\
\hline & до операции & $\begin{array}{c}\text { через } 6 \text { мес после } \\
\text { операции }\end{array}$ & & до операции & $\begin{array}{c}\text { через } 6 \text { мес } \\
\text { после операции }\end{array}$ & \\
\hline ДС & 1,652 & 4,625 & 3 & 1,5 & 4,5 & 3 \\
\hline 4C & 0,5 & 1,75 & 1,25 & 0,5 & 1,75 & 1,25 \\
\hline
\end{tabular}

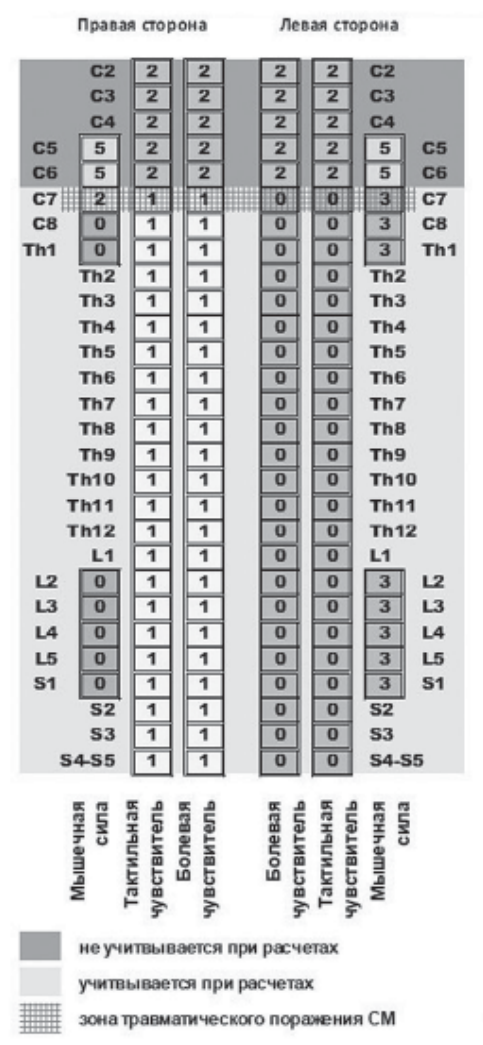

Рис. 3. Схема сегментов СМ, учитываемых при расчете СФА.

Таким образом, у пациента с травмой шейного отдела позвоночника, осложненной повреждением СМ, восстановление ДС составило 48\%, ЧС - 51,3\%, неврологического статуса - 50,3\%; у пациента с травмой нижнегрудного отдела позвоночника, осложненной повреждением СМ, соответственно - 30, 22,3 и 24,7\%.

С целью реализации поставленной задачи оценка проведена у пациентов со сходными по степени выраженности нарушениями функции СМ и динамикой восстановления. Исходно у обоих пациентов ниже уровня поражения СМ сила в левых конечностях оценена в 0 баллов, правых - в 3 балла, после проведенной терапии - соответственно в 4 и 5 баллов. При исследовании чувствительности в обоих пациентов справа отмечалась болевая и тактильная гипестезия ниже уровня поражения, слева - анестезия. После терапии справа зарегистрирована нормальная чувствительность, слева - тактильная гипестезия и нормальная болевая чувствительность.

Принимая во внимание, что клиническая картина у подавляющего большинства пациентов с осложненны- ми повреждениями позвоночника определяется проводниковыми нарушениями СМ на уровне поражения, эффективность терапии в обоих пациентов можно считать идентичной, что в принципе отображается соответствием числовых значений изучаемого признака.

\section{Обсуждение}

Предложенный термин «Динамика восстановления неврологического статуса» достаточно информативен и имеет этимологическое обоснование, так как при его расчете учитывается состояние всех сегментов СM, предусмотренных методикой ISNCSCI, то есть неврологический статус.

Показатель может применяться для индивидуальной оценки динамики течения травматической болезни СМ либо для анализа группы пациентов при условии, что травмирован идентичный по уровню сегмент. Рассчитанное значение фактически демонстрирует, на сколько процентов от абсолютной нормы изменилась функциональная активность СМ в целом.

С целью расчета показателя, характеризующего эффективность проводимой терапии у пациентов с различным уровнем травматического поражения СМ, предлагается учитывать только сегменты, расположенные на уровне и ниже травмы, то есть те, функциональная активность которых была изменена в результате механического воздействия на СМ с последующими сегментарными и проводниковыми нарушениями.

Согласно данной концепции у пациента с травмой шейного отдела позвоночника, осложненной повреждением CM, до операции средняя функциональная активность (СФА) двигательных сегментов будет равняться сумме баллов, полученной при делении суммы баллов C7-S1 слева и справа на количество сегментов, умноженное на количество анализируемых значений в одном сегменте, то есть 26/16 = 1,652 балла.

Таким образом, СФА сегментов, расположенных на уровне и ниже травмы, рассчитывается по формуле:

$$
\begin{gathered}
\text { СФА = сумма баллов анализируемых сегментов/ } \\
\text { количество сегментов } x \\
\text { количество анализируемых значений в одном сегменте. }
\end{gathered}
$$

При анализе ДС количество анализируемых значений в одном сегменте будет 2 (ключевые мышцы слева и справа), ЧС - 4 (тактильная и глубокая чувствительность в сегменте слева и справа).

Значения СФА, расчитанные для обоих пациентов, приведены в табл.3.

Схематически зона сегментов СМ, учитываемых при расчете СФА, у пациента с травмой шейного отдела позвоночника представлена на рис. 3 . 
Как видно из представленных данных, динамика восстановления СФА сегментов СМ на уровне и ниже воздействия травмирующего фактора $(\Delta \mathrm{CФА})$ в обоих пациентов идентична. Исходя из этимологии и учитывая, что в расчет принимаются только сегменты с измененной функциональной активностью, рассчитанный параметр целесообразно характеризовать как динамику восстановления неврологических расстройств.

При проведении расчетов количество анализируемых сегментов СМ в каждом индивидуальном случае должно оставаться постоянным, что позволяет получить наиболее достоверные данные.

Приведенные результаты анализа показывают, что предложенный параметр адекватно отражает динамику регресса функциональных нарушений СМ и может применяться для анализа эффективности терапии у пациентов с различным анатомическим уровнем поражения СМ.

\section{Выводы}

1. Разработаны два критерия оценки динамики состояния функциональной активности СМ у пациентов с осложненной спинальной травмой: «Динамика восстановления неврологического статуса», который отражает изменение функциональной активности СМ в целом и может быть использован для индивидуальной оценки эффективности терапии у пациента, и «Динамика восстановления неврологических расстройств», который характеризует изменение функциональной активности сегментов СМ, расположенных на уровне и ниже травмы, и целесообразен для оценки различных методов лечения у пациентов вне зависимости от анатомического уровня поражения СМ.

2. Продемонстрирована низкая информативность широко используемой системы оценки неврологических расстройств по шкале ASIA.

3. Основным преимуществом предложенных критериев является их цифровое представление, что позволяет выполнять полноценную статистическую обработку полученных результатов с учетом уровня погрешности и статистической достоверности.

4. Разработанная система оценки в случае успешной клинической апробации может быть рекомендована для определения целесообразности и эффективности различных методов хирургической и медикаментозной коррекции при травматических повреждениях СМ.

\section{References}

1. Kirshblum SC, Burns SP, Biering-Sorensen F, Donovan W, Graves $\mathrm{DE}$, Jha $\mathrm{A}$, et al. International standards for neurological classification of spinal cord injury (revised 2011). J Spinal Cord Med. 2011 Nov;34(6):535-46.

2. Kalsi-Ryan S, Beaton D, Curt A, Duff S, Popovic MR, Rudhe C, et al. The Graded Redefined Assessment of Strength Sensibility and Prehension: Reliability and Validity. J Neurotrauma. 2012 Mar 20;29(5):90514.

3. Krassioukov A, Biering-Sørensen F, Donovan W, Kennelly M, Kirshblum S, Krogh K, et al. International standards to document remaining autonomic function after spinal cord injury. J Spinal Cord Med. 2012 Jul 19;35(4):201-10.

4. Kramer JLK, Taylor P, Haefeli J, Blum J, Zariffa J, Curt A, et al. TestRetest Reliability of Contact Heat-Evoked Potentials From Cervical Dermatomes. J Clin Neurophysiol. 2012 Feb;29(1):70-5.

5. McKay WB, Ovechkin A V, Vitaz TW, Terson de Paleville DGL, Harkema SJ. Neurophysiological characterization of motor recovery in acute spinal cord injury. Spinal Cord. 2011 Mar 16;49(3):421-9.

6. King NKK, Savic G, Frankel H, Jamous A, Ellaway PH. Reliability of Cutaneous Electrical Perceptual Threshold in the Assessment of Sensory Perception in Patients with Spinal Cord Injury. J Neurotrauma. 2009 Jul;26(7):1061-8.

7. Miyanji F, Furlan JC, Aarabi B, Arnold PM, Fehlings MG. Acute cervical traumatic spinal cord injury: MR imaging findings correlated with neurologic outcome - prospective study with 100 consecutive patients. Radiology. 2007 Jun;243(3):820-7.

8. Martin AR, Aleksanderek I, Cohen-Adad J, Tarmohamed Z, Tetreault L, Smith N, et al. Translating state-of-the-art spinal cord MRI techniques to clinical use: A systematic review of clinical studies utilizing DTI, MT, MWF, MRS, and fMRI. NeuroImage Clin. 2016;10:192238.

9. Wilson JR, Grossman RG, Frankowski RF, Kiss A, Davis AM, Kulkarni $\mathrm{AV}$, et al. A clinical prediction model for long-term functional outcome after traumatic spinal cord injury based on acute clinical and imaging factors. J Neurotrauma. 2012 Sep;29(13):2263-71.

10. van Hedel HJ, Wirz M, Dietz V. Assessing walking ability in subjects with spinal cord injury: Validity and reliability of 3 walking tests. Arch Phys Med Rehabil. 2005 Feb;86(2):190-6.

11. van Hedel HJA, Wirz M, Curt A. Improving walking assessment in subjects with an incomplete spinal cord injury: responsiveness. Spinal Cord. 2006 Jun 22;44(6):352-6.

12. Wirz M, Müller R, Bastiaenen C. Falls in persons with spinal cord injury: validity and reliability of the Berg Balance Scale. Neurorehabil Neural Repair. 2010 Jan 12;24(1):70-7.

13. Paternostro-Sluga T, Grim-Stieger M, Posch M, Schuhfried O Vacariu G, Mittermaier C, et al. Reliability and validity of the Medical Research Council (MRC) scale and a modified scale for testing muscle strength in patients with radial palsy. J Rehabil Med. 2008 Aug;40(8):665-71.

14. Fehlings MG, Tetreault LA, Wilson JR, Kwon BK, Burns AS, Martin AR, et al. A Clinical Practice Guideline for the Management of Acute Spinal Cord Injury: Introduction, Rationale, and Scope. Glob Spine J. 2017 Sep 5;7(3_suppl):84S-94S. 